\title{
Chronic non-transmural infarction has a delayed recovery of function following revascularization
}

\author{
Martin Ugander ${ }^{1}$, Peter A Cain ${ }^{1}$, Per Johnsson², John Palmer ${ }^{3}$, Håkan Arheden $^{1 *}$
}

\begin{abstract}
Background: The time course of regional functional recovery following revascularization with regards to the presence or absence of infarction is poorly known. We studied the effect of the presence of chronic nontransmural infarction on the time course of recovery of myocardial perfusion and function after elective revascularization.
\end{abstract}

Methods: Eighteen patients (mean age 69, range 52-84, 17 men) prospectively underwent cine magnetic resonance imaging (MRI), delayed contrast enhanced MRI and rest/stress $99 \mathrm{~m}$-Tc-tetrofosmin single photon emission computed tomography (SPECT) before, one and six months after elective coronary artery bypass grafting (CABG) or percutaneous coronary intervention ( $\mathrm{PCl})$.

Results: Dysfunctional myocardial segments ( $n=337 / 864,39 \%)$ were classified according to the presence $(n=$ 164) or absence $(n=173$ ) of infarction. Infarct transmurality in dysfunctional segments was largely non-transmural (transmurality $=31 \pm 22 \%$ ). Quantitative stress perfusion and wall thickening increased at one month in dysfunctional segments without infarction $(p<0.001)$, with no further improvement at six months. Despite improvements in stress perfusion at one month $(p<0.001)$, non-transmural infarction displayed a slower and lesser improvement in wall thickening at one $(p<0.05)$ and six months $(p<0.001)$.

Conclusions: Dysfunctional segments without infarction represent repetitively stunned or hibernating myocardium, and these segments improved both perfusion and function within one month after revascularization with no improvement thereafter. Although dysfunctional segments with non-transmural infarction improved in perfusion at one month, functional recovery was mostly seen between one and six months, possibly reflecting a more severe ischemic burden. These findings may be of value in the clinical assessment of regional functional recovery in the time period after revascularization.

\section{Background}

Revascularization of dysfunctional but viable myocardium in patients with chronic ischemic heart disease (CIHD) may offer both functional improvement of myocardium and prognostic benefit [1]. The pathophysiology underlying the development and recovery of hypofunctioning but viable myocardium in CIHD is not completely understood [2]. Myocardium which is hypofunctioning but viable at rest may represent either hibernating or repetitively stunned myocardium [2]. Therefore, for the purposes of this article, we will use the collective term "dysfunctional but viable myocardium". Dysfunctional myocardial segments have been

\footnotetext{
* Correspondence: hakan.arheden@med.lu.se

'Department of Clinical Physiology, Lund University Hospital, Lund University, Lund, SE-221 85 Sweden
}

shown to improve function immediately post-operatively with no further change 8 days after CABG [3]. This finding indicates that functional recovery begins early. However, others have shown that continued functional recovery is present at follow-up between three and 14 months later [4-9]. Thus, previous studies have shown varying results with regards to the time course of functional recovery following revascularization for dysfunctional segments with or without infarction, and remote myocardium, respectively.

Delayed contrast enhanced magnetic resonance imaging (DE-MRI) has shown to be valuable for predicting regional functional improvement after revascularization [10]. Furthermore, perfusion of dysfunctional but viable myocardium has been shown to improve soon after revascularization [11]. However, it is not known if the

\section{CiolMed Central}


time course of recovery for perfusion and function following revascularization is the same for dysfunctional segments with or without the presence of non-transmural infarction as determined by DE-MRI. Therefore, we sought to quantitatively assess the influence of the presence of non-transmural myocardial infarction on the time course for regional recovery of function by MRI, and perfusion by ${ }^{99 \mathrm{~m}} \mathrm{Tc}$-tetrofosmin single photon emission computed tomography (SPECT) over a six month period after revascularization.

\section{Methods}

\section{Study population}

The study was approved by the ethics committee on human research at Lund University Hospital. All patients provided written informed consent. Patients were prospectively enrolled between December, 2001 and May, 2005. The inclusion criterion was clinical selection for first time elective revascularization by coronary artery bypass grafting (CABG) or percutaneous coronary intervention (PCI). Following revascularization, changes in medication were determined by the caring physician. Patients were imaged with cardiac MRI and rest/stress SPECT prior to revascularization and one and six months after revascularization. A schematic diagram of the timeline of the study is presented in Figure 1. Exclusion criteria were valvular surgery in adjunct to revascularization, acute coronary syndrome during the course of the study, New York Heart Association functional class IV, absence of sinus rhythm, claustrophobia or contraindications for MRI.

\section{MR Imaging}

Left ventricular function and viability were imaged in the short-axis plane during breath hold using a 1.5T system (Magnetom Vision, Siemens, Erlangen, Germany or Intera CV, Philips, Best, the Netherlands). Global and regional function was imaged using an ECG-triggered cine gradient echo sequence (Siemens, resolution $1.6 \times$ $1.6 \times 8 \mathrm{~mm}$, gap $2 \mathrm{~mm}$, temporal resolution $50 \mathrm{~ms}$ ) or a retrospectively vector-ECG-triggered cine steady-state free precession sequence (Philips, spatial resolution 1.25 $\times 1.25 \times 8 \mathrm{~mm}$, gap $0 \mathrm{~mm}$, temporal resolution $33 \mathrm{~ms}$, SENSE factor 2). Infarct imaging was undertaken using a segmented inversion recovery turbo fast low-angle shot sequence [12] in either 2D or 3D (2D: Siemens, acquisition every other heart beat, resolution $1.6 \times 1.6 \times$ $8 \mathrm{~mm}$, gap $2 \mathrm{~mm}, 3 \mathrm{D}$ : Philips, acquisition every heart beat, $1.6 \times 1.6 \times 8 \mathrm{~mm}$, gap $0 \mathrm{~mm}$, inversion time set to null normal myocardium). Imaging was performed 15 20 minutes after intravenous injection of $0.2 \mathrm{mmol} / \mathrm{kg}$ body weight of an extracellular contrast agent (Magnevist $^{\circ}$, gadopentetate dimeglumine, Gd-DTPA, Schering Nordiska AB, Järfälla, Sweden). This approach has been shown to enhance non-viable myocardium [13] due to an increased tissue distribution volume of Gd-DTPA in non-viable regions [14].

\section{SPECT Imaging}

Rest and stress SPECT imaging were performed on separate days, 30 minutes after intravenous injection of a body weight adjusted dose (500-700 MBq) of ${ }^{99 \mathrm{~m}} \mathrm{Tc}-$ tetrofosmin (Amersham Health, Buckinghamshire, UK). The same dose was used for rest and stress. For stress imaging, $5 \mathrm{mg} / \mathrm{ml}$ adenosin (Adenosin Item ${ }^{\circ}$, Item Development AB, Stocksund, Sweden) was infused at a rate of $140 \mu \mathrm{g} / \mathrm{kg} / \mathrm{min}$ for 3 minutes before tracer injection, and continued for 2 minutes following injection. For those instances where a subject mistakenly had consumed caffeine in the 24 hours prior to stress imaging, exercise stress was performed using a bicycle ergometer with a minimum required increase of $85 \%$ of the maximum predicted heart rate. For both rest and stress imaging, the subject was imaged in the supine position with a dual head camera (ADAC Vertex, Milpitas, California, USA) using standard methods [15].

\section{Image Analysis}

All studies were quantitatively analyzed using a 48-segment model with 4 midventricular short-axis slices and 12 segments per slice. All images were analyzed using software developed in-house, including the freely available software Segment version $1.661 \mathrm{http}: / /$ segment.heiberg.se [16], as described previously in detail [17]. In summary, the endocardial and epicardial borders of

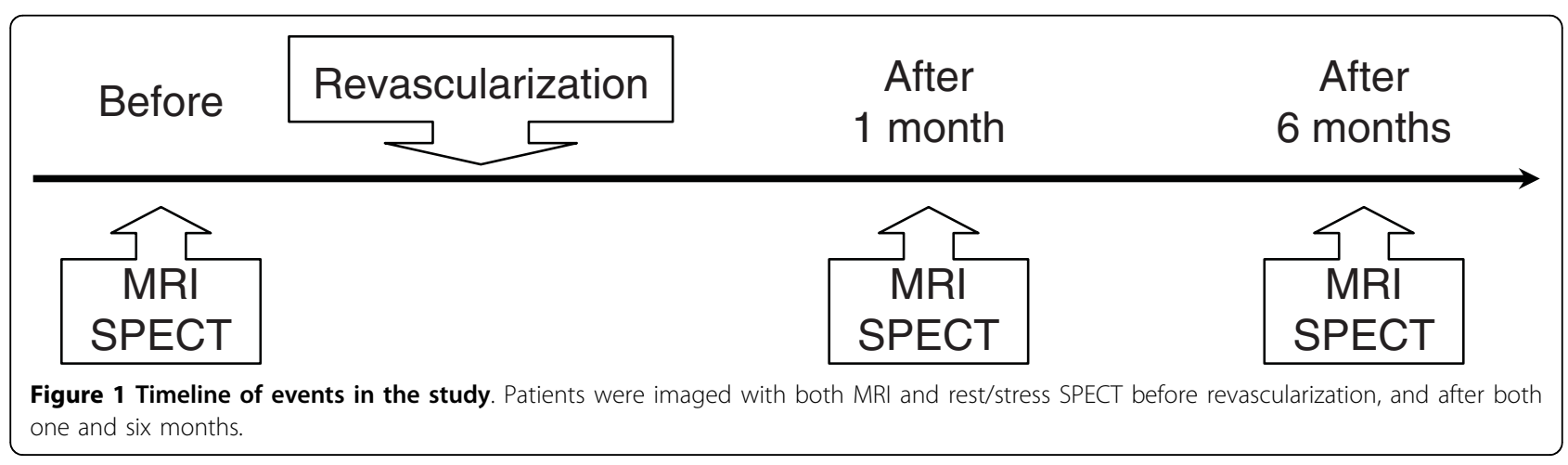


short axis cine MR images were manually delineated in end diastole and end systole for determination of wall thickening. The endocardial and epicardial borders, and the border of the hyperenhanced region of infarction in DE-MRI were manually delineated for determination of infarct transmurality. Infarct transmurality was defined as the transmural extent of infarction divided by the total transmural wall thickness. Semi-automatic quantification of wall thickening from MRI, infarction from DEMRI, and both rest and stress perfusion from SPECT was performed along radial profiles from the centroid of the left ventricular lumen at every other degree, yielding 180 measurements per short axis slice. For any given parameter, measurements were averaged over 30 degrees to obtain values for the 12 -segment per slice model. The four slices analyzed for all subjects were either 3.0, 4.0, 5.0 and $6.0 \mathrm{~cm}$ from the apex (Siemens) or 3.2, 4.0, 4.8 and $5.6 \mathrm{~cm}$ (Philips) from the apex, respectively. Slices closest to the base and apex were excluded to minimize errors in assessment of wall thickness introduced by the partial volume effect and atrioventricular plane movement, respectively. Dysfunction was defined as segments with wall thickening less than 30\% [18]. For MR images, the apex was defined at the time of imaging by commencing the short axis imaging with an apical slice that encompassed the apical tip of the heart. For SPECT, the apex was defined manually in a vertical long axis image, after which contiguous short axis slices were reconstructed. Slice thickness for reconstructed images were $10 \mathrm{~mm}$ or $8 \mathrm{~mm}$ for patients imaged by MR using Siemens or Philips scanners, respectively. See Figure 2.

\section{Statistics}

Time course data are presented as mean \pm SEM. The number of days between imaging and revascularization are presented as median and interquartile range (IQR), defined as the range between the $25^{\text {th }}$ and $75^{\text {th }}$ quartile of the data. All other data are presented as mean \pm SD unless stated otherwise. Variations between data were tested by paired t-test and independent $t$-test as appropriate. $\mathrm{P}<0.05$ was considered statistically significant. Power analysis was calculated retrospectively to determine the difference in wall thickening which one could detect with a power of 0.90 using $\mathrm{p}<0.05$ (two-tailed), a measurement variability (SD) of $1 \mathrm{~mm}$, and given the number of myocardial segments involved in the comparison.

\section{Results}

Patient characteristics including times of imaging in relation to revascularization and baseline medications are described in Table 1. LVEF was unchanged one month and at six months following revascularization (44 $\pm 12 \%, \mathrm{p}=0.253$ and $47 \pm 13 \%, \mathrm{p}=0.225)$. Upon discharge following revascularization, all patients' medications were unchanged or dosages had been reduced except for the following. One patient had an increase in the dosage of both beta-blockers and diuretics. Five separate patients had either an increase in beta-blockers, angiotensin converting enzyme inhibitors or diuretics, respectively. No patient experienced perioperative infarction or died during the study. Two patients were included but lost to follow-up, and therefore not included in the analysis.

\section{Delayed enhancement imaging prior to CABG}

DE-MRI was possible in all patients resulting in $864 \mathrm{seg-}$ ments for analysis. Myocardial segments that were dysfunctional before revascularization $(n=337,39 \%)$ were classified according to the presence $(n=164)$ or absence $(n=173)$ of infarction. Figure 3 shows the distribution of infarct transmuralities for segments that were dysfunctional and segments that were not dysfunctional prior to revascularization. Among dysfunctional segments with infarction, infarct transmurality was $31 \pm$ $22 \%$, range $1-87 \%$.

\section{Time course of regional function}

Retrospective power analysis showed that given a comparison of 164 myocardial segments, there was a power of 0.90 for detecting a change in wall thickening of 0.25 $\mathrm{mm}$. Figure $4 \mathrm{~A}$ shows the time course of regional wall thickening for segments that were dysfunctional before surgery. Non-infarcted segments increased in function at one month $(\mathrm{p}<0.001)$ with no change thereafter $(\mathrm{p}=$ $0.104)$. Segments with infarction, however, showed improvement in function at one month $(\mathrm{p}=0.013)$, but also improvement in function between one and six months $(\mathrm{p}<0.001)$. Figure $4 \mathrm{~B}$ shows that segments with no baseline dysfunction showed a decrease in wall thickening at one month regardless of presence of infarction $(\mathrm{p}<0.001)$. Among these segments, only those without infarction showed a significant increase in function between one and six months $(p<0.001)$.

Time course of regional perfusion

\section{Stress Perfusion}

Figure $4 \mathrm{C}$ shows the time course of quantitative stress perfusion for segments that were dysfunctional before surgery. Segments with and without infarction differed from each other in stress perfusion at all time points ( $p$ $<0.001)$. Both groups showed a similar time course of improvement at one month $(\mathrm{p}<0.001)$ and no further improvement at six months $(\mathrm{p}=0.366$ and $\mathrm{p}=0.965)$. Figure 4D shows that in segments with no baseline dysfunction both with and without infarction, stress perfusion increased mostly at one month $(\mathrm{p}<0.001$ and $\mathrm{p}=$ $0.002)$, but also a six months ( $\mathrm{p}=0.042$ and $\mathrm{p}<0.001)$.

\section{Rest Perfusion}

Figure $4 \mathrm{E}$ shows the time course of rest perfusion for segments that were dysfunctional before surgery. Both groups differed in rest perfusion from each other at all 


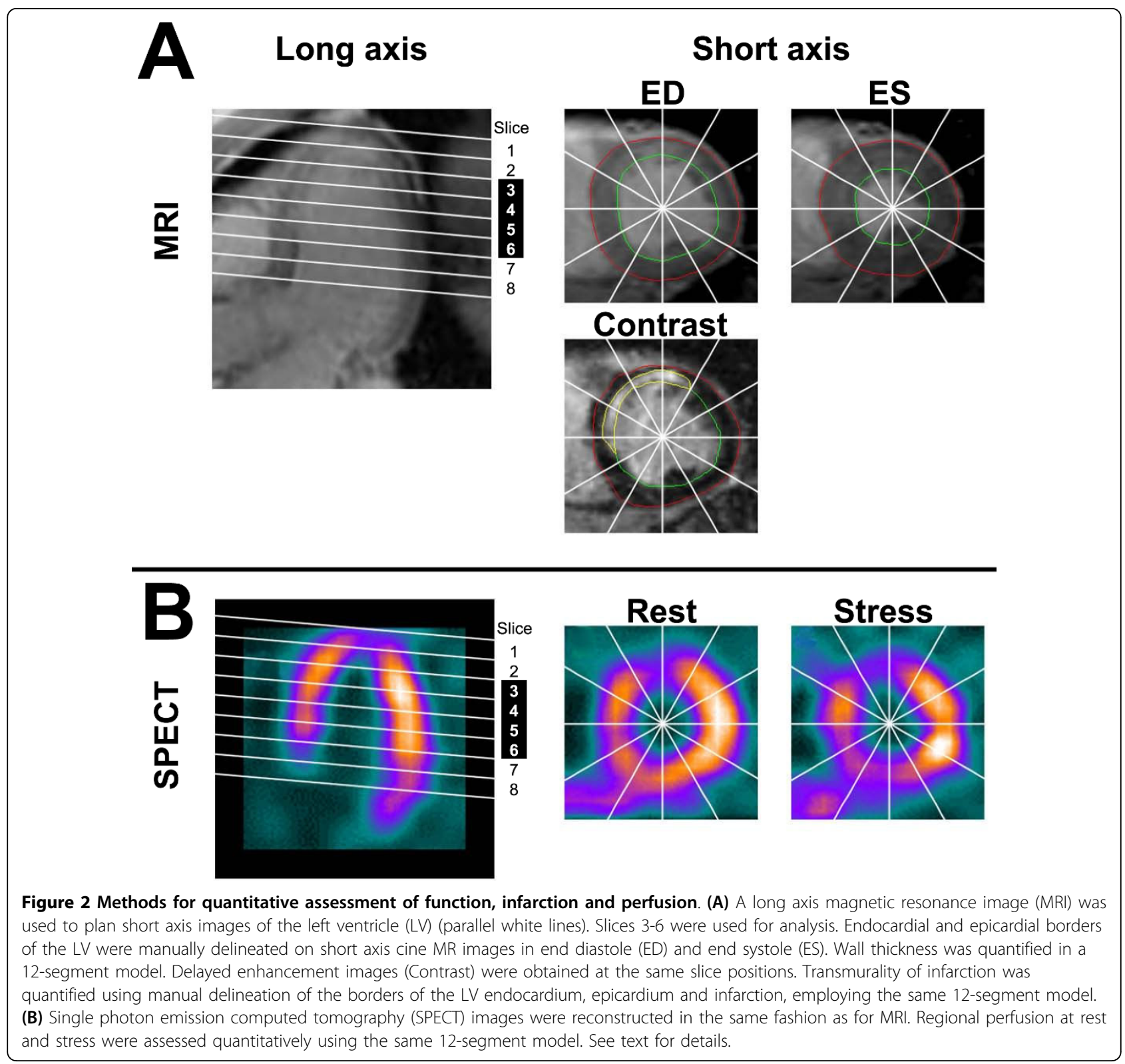

time points $(\mathrm{p}<0.001)$. Only non-infarcted segments improved in resting perfusion between baseline and one month $(\mathrm{p}=0.010)$ and six months $(\mathrm{p}=0.002)$. Figure $4 \mathrm{~F}$ shows that in segments with no baseline dysfunction and without infarction, rest perfusion increased at both one month $(\mathrm{p}=0.023)$ and six months $(\mathrm{p}<0.001)$, while segments with infarction remained unchanged at both time points $(\mathrm{p}=0.539$ and $\mathrm{p}=0.348)$.

\section{Perfusion difference}

Figure 4G shows the time course of the quantitative difference between rest and stress perfusion for segments that were dysfunctional before surgery. The groups only differed significantly from each other at baseline $(p=0.035)$. Both segments with and without infarction decreased significantly between baseline and one month $(\mathrm{p}=0.008$ and $p<0.001$ ) with only non-infarcted segments showing a change between one and six months $(p=0.025)$. Figure $4 \mathrm{H}$ shows that among segments with no baseline dysfunction, only those with infarction were relieved of their perfusion difference at one month $(\mathrm{p}=0.001)$.

\section{Discussion}

To our knowledge, this is the first study to have serially and quantitatively examined the time course after revascularization of regional recovery of perfusion and function according to presence of infarction by DE-MRI. The major findings can be summarized as follows. Dysfunctional segments without infarction gained the 
Table 1 Patient characteristics

\begin{tabular}{|c|c|}
\hline Mean age, $y$ & 69 (range 53-84) \\
\hline Male, n (\%) & $17(94)$ \\
\hline Caucasian race, n (\%) & $18(100)$ \\
\hline 1-vessel disease, $\mathrm{n}(\%)$ & $3(17)$ \\
\hline 2-vessel disease, $\mathrm{n}(\%)$ & $8(44)$ \\
\hline 3-vessel disease, n (\%) & $7(39)$ \\
\hline Diabetes, n (\%) & $7(39)$ \\
\hline Baseline LVEF*, \% & $47 \pm 11$ \\
\hline \multicolumn{2}{|l|}{ Baseline medication with... } \\
\hline Beta-blocker, n (\%) & $15(83)$ \\
\hline Angiotensin converting enzyme inhibitor, n (\%) & $10(56)$ \\
\hline Diuretic, n (\%) & $5(28)$ \\
\hline Baseline MR, median days from revascularization & $-4(\mathrm{QQR}-7$ to -2$)$ \\
\hline Baseline rest SPECT, median days from revascularization & $-4(\mathrm{IQR}-7$ to -4$)$ \\
\hline Baseline stress SPECT, median days from revascularization & $-1(\mathrm{QQR}-6$ to -1$)$ \\
\hline One month MR, median days from revascularization & 35 (IQR 29 to 42$)$ \\
\hline One month SPECT, median days from revascularization & 31 (IQR 28 to 38) \\
\hline One month SPECT, median days from revascularization & 33 (IQR 29 to 41$)$ \\
\hline Six month MR, median days from revascularization & 196 (IQR 187 to 207) \\
\hline Six month SPECT, median days from revascularization & 196 (IQR 187 to 207) \\
\hline Six month SPECT, median days from revascularization & 197 (IQR 188 to 208) \\
\hline
\end{tabular}

* denotes that LVEF data was not available for one patient due to insufficient number of MR images. IQR = interquartile range.

greatest improvement in both perfusion and function within one month after revascularization. In contrast, segments with infarction, which was predominantly $<50 \%$ transmural, showed a slower functional recovery which was obvious first at six months, despite improvements in perfusion already at one month.

\section{Time course of recovery of regional function}

Several studies have examined functional recovery at single time points following revascularization based on the extent of infarction assessed by DE-MRI $[10,19,20]$. Studies using echocardiography and scintigraphic techniques have demonstrated that the time course of regional functional recovery following $C A B G$ begins immediately following revascularization and may continue up to between three and 14 months after surgery [3-9]. For example, one study showed no functional improvement in segments with either non-transmural or transmural infarction as determined by ${ }^{18} \mathrm{~F}$-2-deoxyglucose (FDG) uptake using SPECT [8]. Yet, segments with reduced perfusion but no sign of infarction using FDG improved function both three and 14 months after revascularization. The discrepancy in the time course of functional recovery between previous results and ours may be due to the limited spatial resolution

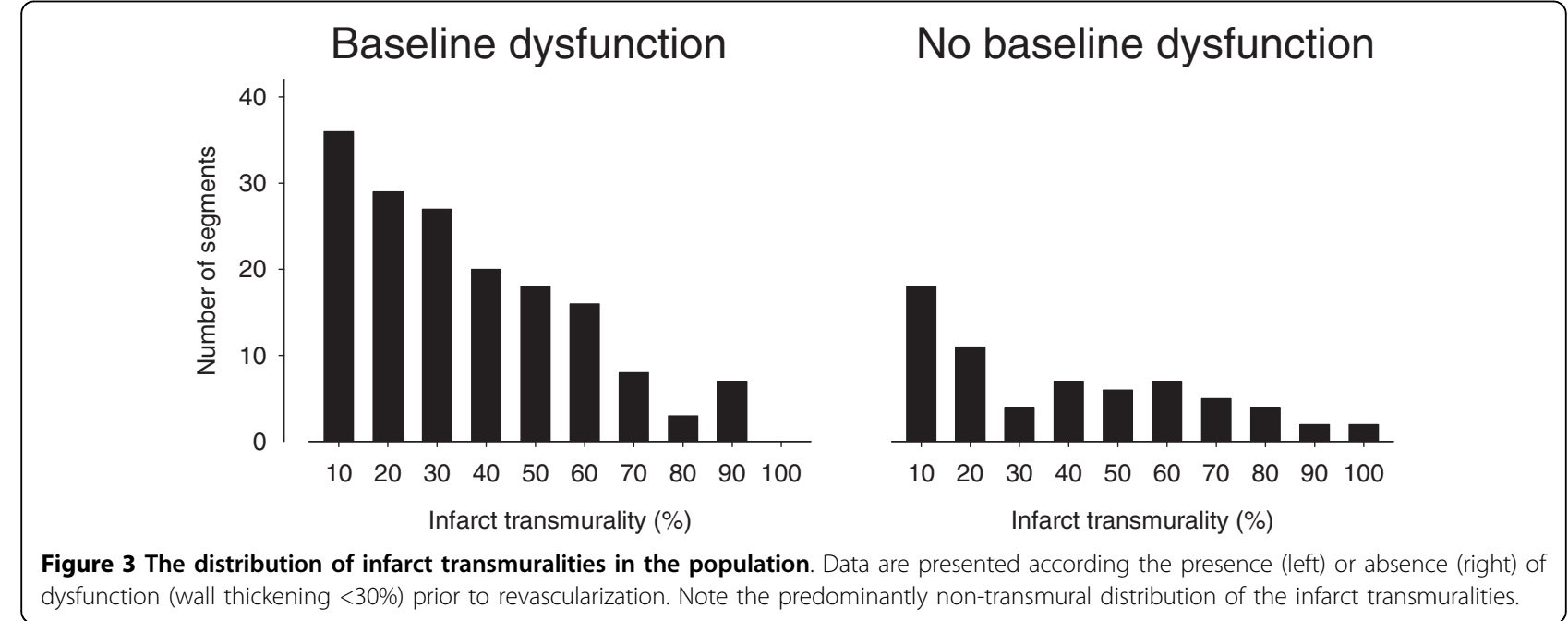

No baseline dysfunction 


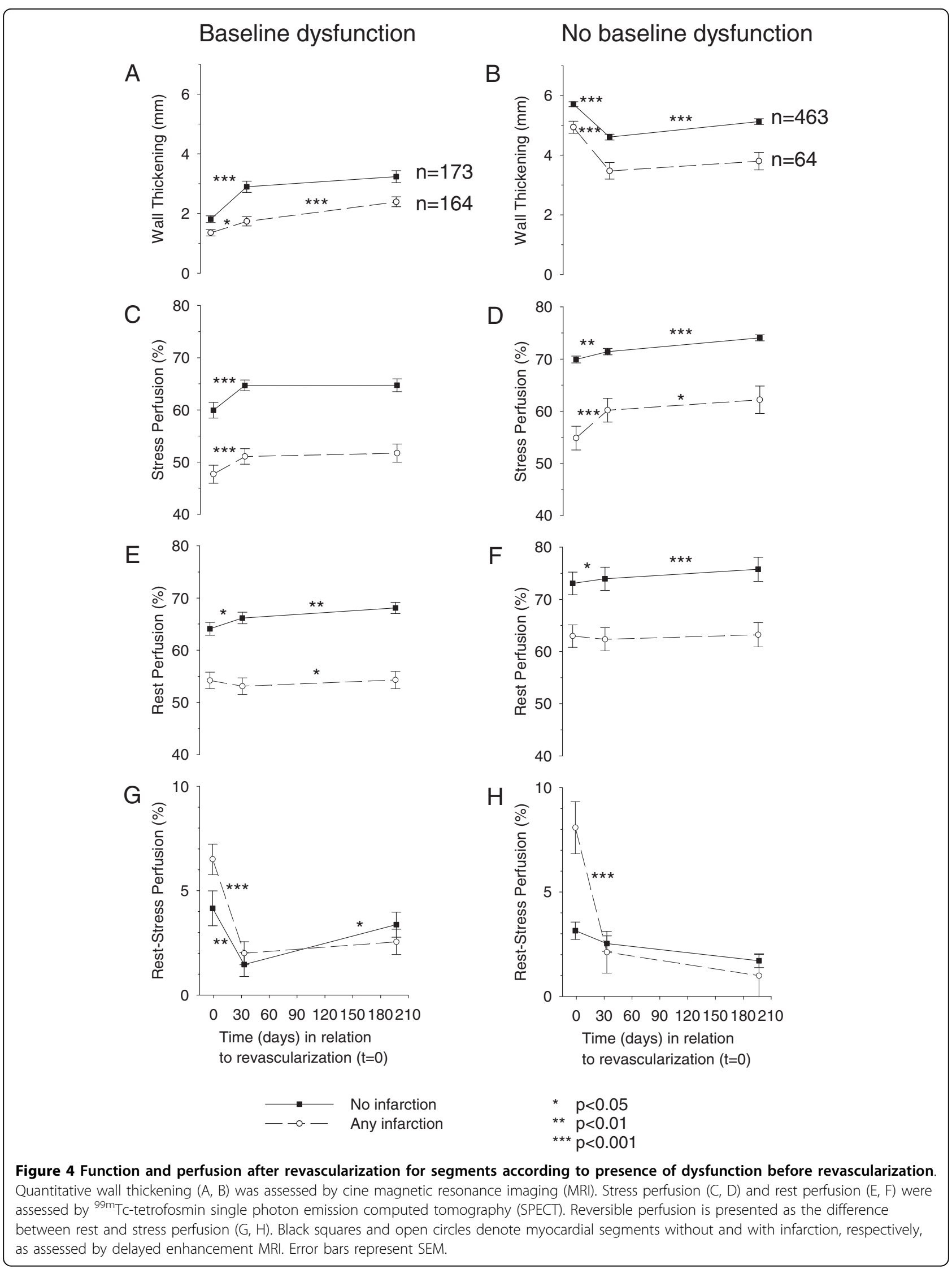


of SPECT for defining the presence and transmurality of infarction in previous studies, while we used DEMRI. Similar to our findings, it was recently shown that the time course of regional functional recovery following revascularization increased with the amount of regional infarction as determined by DE-MRI [21]. However, that study did not assess perfusion, as in the current study.

Late improvements in function assessed visually by echocardiography have been associated with pre-operative histological and scintigraphic measures of ischemic burden, defined as a prolonged duration, greater extent or greater severity of ischemia [4-9]. Such histological measures of ischemic burden have been shown to independently correlate to both scintigraphic findings [22] and an increased duration of ischemia resulting in a delayed functional recovery [23]. Therefore, one potential explanation for our findings of the difference in time course of functional recovery between segments with and without infarction may be a more severe degree of ischemic burden in the viable portion of the segments with infarction. This is supported by our finding of a greater initial perfusion difference in these segments. Alternatively, changes in afterload or compensatory hypertrophy of viable myocardium following revascularization may have contributed to improved regional function, but this was not studied.

Surprisingly, segments without baseline dysfunction showed a reduction in wall thickening at one month regardless of presence of infarction. The reason for this pattern of remodelling is not known. Although we found changes in regional function in different regions within the left ventricle, we found no change in net global function (ejection fraction) following revascularization in our population. Similar to our findings, stent revascularization of chronic total coronary occlusion has been shown to increase wall thickening in dysfunctional segments, but decrease wall thickening in remote segments, with no net change in ejection fraction [24]. Also, ventriculography has been used to report improved regional wall motion in hypokinetic segments but also simultaneous deterioration in wall motion in initially normokinetic segments [25]. Our findings of simultaneous improvement and deterioration of regional function in segments of different baseline functional status seems reasonable considering the lack of change in LVEF in our population. Thus, the improved outcome in revascularized patients [1], despite sometimes limited improvements in ejection fraction, may be caused not only by a relief of ischemia in ischemic but viable regions but also a relief of compensatory hyperfunction in remote and normally functioning myocardium.

\section{Time course of recovery of regional perfusion Rest Perfusion}

The current study agrees with previous findings that scintigraphically assessed regional perfusion at rest improves soon after surgical and percutaneous revascularization [11].

\section{Stress Perfusion}

The current study also agrees with previous findings showing an improvement in scintigraphically assessed stress perfusion at single examinations after revascularization. This has been shown soon after percutaneous revascularization [26] and both 2-5 weeks [4] and 3-4 months [27] after surgical revascularization. Manyari et al [28], showed a progressive improvement in stress perfusion at 9 days and 3 months, but not 6 months following percutaneous revascularization. Taken together with our findings, it appears that incremental improvement in perfusion can be identified no later than 3 months after revascularization.

\section{Perfusion difference}

The difference between rest and stress perfusion may represent stress induced ischemia. Previous studies have examined the extent of reversible perfusion defects that exceed a certain severity cut-off value [29]. To our knowledge, this is the first study to examine the severity of reversibility of perfusion for given segments in this manner. Our results for both perfusion and function are consistent with the proposed mechanism that a greater ischemic burden may be involved in the delayed functional recovery of segments with infarction.

Infarct transmurality and baseline function

When comparing the distribution of infarct transmurality in relation to baseline dysfunction, the current study showed, as expected, that there was a greater prevalence of infarction among segments with baseline dysfunction. The distribution of infarction in the population was largely non-transmural. However, it is noteworthy that there existed segments without baseline dysfunction which had as much as $100 \%$ infarct transmurality. This may seem counterintuitive. However, this represents very few myocardial segments $(n=2)$, and can be explained by tethering to adjacent segments. For example, it has been shown that myocardial segmental function is influenced moreso by the function of neighbouring segments than by infarct transmurality as such [30].

\section{Limitations of the Study}

The number of patients is limited, however, power analysis showed that the study was adequately powered to assess differences in regional function. The number of segments with different degrees of transmurality did not permit an adequate separate analysis for infarction of different transmuralities. Although care was taken to 
achieve maximal alignment of slices between modalities and time points, misalignment due to fundamental differences in the imaging modalities is also a potential limitation.

\section{Conclusions}

This study has demonstrated that dysfunctional segments without infarction, also known as hibernating or repetitively stunned myocardium, improved in both perfusion and function within one month after revascularization. Although segments with predominantly nontransmural infarction improved in rest and stress perfusion at one month, functional recovery was slower and clearly seen first at six months. Despite lack of change in ejection fraction, remote myocardium showed a decreased wall thickening suggesting relief of compensatory contractile work. This may be of value in the clinical assessment of regional function and suggests that evaluation of the coronary revascularization procedure with regards to regional function should be performed first 6 months or later after the intervention in patients with previous infarction. Further studies are warranted to elucidate the pathophysiological mechanism behind these findings.

\section{Abbreviations \\ CABG: coronary artery bypass surgery; CIHD: chronic ischemic heart; DE: delayed enhancement; ECG: electrocardiogram; FDG: ${ }^{18} \mathrm{~F}$-2-deoxyglucose; Gd-DTPA: gadolinium diethylene triamine pentaacetic acid; IQR: interquartile range; MRI: magnetic resonance imaging; $\mathrm{PCl}$ : percutaneous coronary intervention; SPECT: single photon emission computed tomography; Tc: technetium \\ Acknowledgements \\ The authors wish to acknowledge nurses Kristina Engels, RN and Carin Paulsson, RN for help with patient recruiting and technicians Ann-Helen Arvidsson and Christel Carlander for help with patient administration and imaging. This study was funded in part by the Swedish National Research Council, Swedish Heart Lung Foundation, Lund University Faculty of Medicine and the Region of Scania.}

\section{Author details \\ 'Department of Clinical Physiology, Lund University Hospital, Lund University, Lund, SE-221 85 Sweden. 'Department of Cardiothoracic Surgery, Lund University Hospital, Lund University, Lund, SE-221 85 Sweden. ${ }^{3}$ Department of Medical Radiation Physics, Lund University Hospital, Lund University, Lund, SE-221 85 Sweden.}

\section{Authors' contributions}

$\mathrm{MU}$ participated in design, performed data acquisition and image analysis, data analysis and wrote the manuscript. PC participated in design, data analysis, and writing the manuscript. JP and PJ participated in design and revised the manuscript for important intellectual content. HA conceived of the study, designed the study and participated in data analysis and writing the manuscript. All authors read and approved the final manuscript.

\section{Competing interests}

The authors declare that they have no competing interests.

Received: 12 May 2009

Accepted: 18 January 2010 Published: 18 January 2010

\section{References}

1. Lee KS, Marwick TH, Cook SA, Go RT, Fix JS, James KB, Sapp SK, Maclntyre WJ, Thomas JD: Prognosis of patients with left ventricular dysfunction, with and without viable myocardium after myocardial infarction. Relative efficacy of medical therapy and revascularization. Circulation 1994, 90:2687-2694.

2. Kloner RA, Bolli R, Marban E, Reinlib L, Braunwald E: Medical and cellular implications of stunning, hibernation, and preconditioning: an NHLBI workshop. Circulation 1998, 97:1848-1867.

3. Topol EJ, Weiss JL, Guzman PA, Dorsey-Lima S, Blanck TJ, Humphrey LS, Baumgartner WA, Flaherty JT, Reitz BA: Immediate improvement of dysfunctional myocardial segments after coronary revascularization: detection by intraoperative transesophageal echocardiography. J Am Coll Cardiol 1984, 4:1123-1134.

4. Takeishi Y, Tono-oka I, Kubota I, Ikeda K, Masakane I, Chiba J, Abe S, Tsuiki K, Komatani A, Yamaguchi I: Functional recovery of hibernating myocardium after coronary bypass surgery: does it coincide with improvement in perfusion?. Am Heart J 1991, 122:665-670.

5. Elsasser A, Schlepper M, Klovekorn WP, Cai WJ, Zimmermann R, Muller KD, Strasser R, Kostin S, Gagel C, Munkel B, et al: Hibernating myocardium: an incomplete adaptation to ischemia. Circulation 1997, 96:2920-2931.

6. Haas F, Augustin N, Holper K, Wottke M, Haehnel C, Nekolla S, Meisner H, Lange R, Schwaiger M: Time course and extent of improvement of dysfunctioning myocardium in patients with coronary artery disease and severely depressed left ventricular function after revascularization: correlation with positron emission tomographic findings. J Am Coll Cardiol 2000, 36:1927-1934

7. Haas F, Jennen L, Heinzmann U, Augustin N, Wottke M, Schwaiger M, Lange R: Ischemically compromised myocardium displays different timecourses of functional recovery: correlation with morphological alterations?. Eur J Cardiothorac Surg 2001, 20:290-298.

8. Bax JJ, Visser FC, Poldermans D, Elhendy A, Cornel JH, Boersma E, van Lingen A, Fioretti PM, Visser CA: Time course of functional recovery of stunned and hibernating segments after surgical revascularization. Circulation 2001, 104:1314-318.

9. Vanoverschelde $\mathrm{L}$, Depre C, Gerber BL, Borgers M, Wijns W, Robert A Dion $R$, Melin JA: Time course of functional recovery after coronary artery bypass graft surgery in patients with chronic left ventricular ischemic dysfunction. Am J Cardiol 2000, 85:1432-1439.

10. Kim RJ, Wu E, Rafael A, Chen EL, Parker MA, Simonetti O, Klocke FJ, Bonow RO, Judd RM: The use of contrast-enhanced magnetic resonance imaging to identify reversible myocardial dysfunction. N Engl J Med 2000, 343:1445-1453.

11. Altehoefer C, vom Dahl J, Messmer BJ, Hanrath P, Buell U: Fate of the resting perfusion defect as assessed with technetium-99 m methoxyisobutyl-isonitrile single-photon emission computed tomography after successful revascularization in patients with healed myocardial infarction. Am J Cardiol 1996, 77:88-92.

12. Simonetti OP, Kim RJ, Fieno DS, Hillenbrand HB, Wu E, Bundy JM, Finn JP, Judd RM: An improved MR imaging technique for the visualization of myocardial infarction. Radiology 2001, 218:215-223.

13. Kim RJ, Fieno DS, Parrish TB, Harris K, Chen EL, Simonetti O, Bundy J, Finn JP, Klocke FJ, Judd RM: Relationship of MRI delayed contrast enhancement to irreversible injury, infarct age, and contractile function. Circulation 1999, 100:1992-2002.

14. Tong CY, Prato FS, Wisenberg G, Lee TY, Carroll E, Sandler D, Wills J, Drost $D$ : Measurement of the extraction efficiency and distribution volume for Gd-DTPA in normal and diseased canine myocardium. Magn Reson Med 1993, 30:337-346.

15. AutoQuant User's Manual 9201-02225D-ENG, Rev A ADAC Laboratories 2000, Appendix B1-B2.

16. Heiberg E, Sjogren J, Ugander M, Carlsson M, Engblom H, Arheden $H$ : Design and validation of Segment - freely available software for cardiovascular image analysis. BMC Med Imaging 2010, 10:1.

17. Cain PA, Ugander M, Palmer J, Carlsson M, Heiberg E, Arheden H: Quantitative polar representation of left ventricular myocardial perfusion, function and viability using SPECT and cardiac magnetic resonance: initial results. Clin Physiol Funct Imaging 2005, 25:215-222.

18. Chan J, Wahi S, Cain P, Marwick TH: Anatomical M-mode: A novel technique for the quantitative evaluation of regional wall motion 
analysis during dobutamine echocardiography. Int J Card Imaging 2000, 16:247-255.

19. Knuesel PR, Nanz D, Wyss C, Buechi M, Kaufmann PA, von Schulthess GK, Luscher TF, Schwitter J: Characterization of dysfunctional myocardium by positron emission tomography and magnetic resonance: relation to functional outcome after revascularization. Circulation 2003, 108:1095-1100.

20. Schvartzman PR, Srichai MB, Grimm RA, Obuchowski NA, Hammer DF, McCarthy PM, Kasper JM, White RD: Nonstress delayed-enhancement magnetic resonance imaging of the myocardium predicts improvement of function after revascularization for chronic ischemic heart disease with left ventricular dysfunction. Am Heart J 2003, 146:535-541.

21. Bondarenko O, Beek AM, Twisk JW, Visser CA, van Rossum AC: Time course of functional recovery after revascularization of hibernating myocardium: a contrast-enhanced cardiovascular magnetic resonance study. Eur Heart J 2008, 29:2000-2005.

22. Maes A, Flameng W, Nuyts J, Borgers M, Shivalkar B, Ausma J, Bormans G, Schiepers C, De Roo M, Mortelmans L: Histological alterations in chronically hypoperfused myocardium. Correlation with PET findings. Circulation 1994, 90:735-745.

23. Schwarz ER, Schoendube FA, Kostin S, Schmiedtke N, Schulz G, Buell U, Messmer BJ, Morrison J, Hanrath P, vom Dahl J: Prolonged myocardial hibernation exacerbates cardiomyocyte degeneration and impairs recovery of function after revascularization. J Am Coll Cardiol 1998, 31:1018-1026.

24. Baks T, van Geuns RJ, Duncker DJ, Cademartiri F, Mollet NR, Krestin GP, Serruys PW, de Feyter PJ: Prediction of left ventricular function after drug-eluting stent implantation for chronic total coronary occlusions. J Am Coll Cardiol 2006, 47:721-725.

25. Jeppson GM, Clayton PD, Blair TJ, Liddle HV, Jensen RL, Klausner SC: Changes in left ventricular wall motion after coronary artery bypass surgery: signal or noise?. Circulation 1981, 64:945-951.

26. Carvalho PA, Vekshtein VI, Tumeh SS, Alajaji G, Nagel JS, Kirshenbaum J, DeSisto WC, Holman BL: Tc-99 m MIBI SPECT in the assessment of myocardial reperfusion after percutaneous transluminal coronary angioplasty. Clin Nucl Med 1991, 16:819-825.

27. Paluszkiewicz L, Kwinecki P, Jemielity M, Szyszka A, Dyszkiewicz W, Cieslinski A: Myocardial perfusion correlates with improvement of systolic function of the left ventricle after CABG. Dobutamine echocardiography and Tc-99 m-MIBI SPECT study. Eur J Cardiothorac Surg 2002, 21:32-35.

28. Manyari DE, Knudtson M, Kloiber R, Roth D: Sequential thallium-201 myocardial perfusion studies after successful percutaneous transluminal coronary artery angioplasty: delayed resolution of exercise-induced scintigraphic abnormalities. Circulation 1988, 77:86-95.

29. Kubo S, Tadamura E, Kudoh T, Inubushi M, Ikeda T, Koshiji T, Nishimura K, Komeda M, Tamaki N, Konishi J: Assessment of the effect of revascularization early after CABG using ECG-gated perfusion singlephoton emission tomography. Eur J Nucl Med 2001, 28:230-239.

30. Ugander M, Cain PA, Perron A, Hedstrom E, Arheden H: Infarct transmurality and adjacent segmental function as determinants of wall thickening in revascularized chronic ischemic heart disease. Clin Physiol Funct Imaging 2005, 25:209-214.

\section{Pre-publication history}

The pre-publication history for this paper can be accessed here:http://www. biomedcentral.com/1471-2261/10/4/prepub

doi:10.1186/1471-2261-10-4

Cite this article as: Ugander et al:: Chronic non-transmural infarction has a delayed recovery of function following revascularization. $B M C$

Cardiovascular Disorders 2010 10:4. 